\title{
Mild cognitive impairment, degenerative and vascular dementia as predictors of intra-hospital, short- and long-term mortality in the oldest old
}

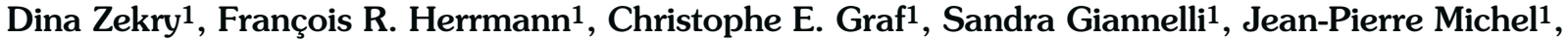 \\ Gabriel Gold ${ }^{1}$ and Karl-Heinz Krause ${ }^{2}$ \\ 1Rehabilitation and Geriatrics Department, Geneva University Hospitals and University of Geneva, Thônex, \\ 2Pathology and Immunology Department, Geneva University, Geneva, Switzerland
}

ABSTRACT. Background and aims: The relative weight of various etiologies of dementia and mild cognitive impairment (MCI) as predictors of intra-hospital, short- and long-term mortality in very old acutely ill patients suffering from multiple comorbid conditions remains unclear. We investigated intra-hospital, 1and 5-year mortality risk associated with dementia and its various etiologies in a very old population after discharge from acute care. Methods: Prospective cohort study of 444 patients (mean age 85 years; $74 \%$ female) discharged from the acute geriatric unit of Geneva University Hospital. On admission, each subject underwent standardized evaluation of cognitive and comorbid conditions. Patients were followed yearly by the same team. Predictive variables were age, sex, cognitive diagnosis, dementia etiology and severity. Survival during hospitalization, at 1- and 5-year follow-ups was the outcome of interest evaluated with Cox proportional hazard models. Results: Two hundred and six patients were cognitively normal, 48 had MCI, and 190 had dementia: of these, there were 75 cases of Alzheimer's disease (AD), 20 of vascular dementia (VaD), 82 of mixed dementia (MD) and 13 of other types of dementia. The groups compared were statistically similar in age, sex, education level and comorbidity score. After 5 years of follow-up, $60 \%$ of the patients had died. Regarding intra-hospital mortality, none of the predictive variables was associated with mortality. MCI, $A D$ and $M D$ were not predictive of short- or long-term mortality. Features significantly associated with reduced survival at 1 and 5 years were being older, male, and having vascular or severe dementia. When all the variables were added in the multiple model, the dementia effect completely dis- appeared. Conclusions: Dementia (all etiologies) is not predictive of mortality. The observed VaD effect is probably linked to cardiovascular risk comorbidities: hypertension, stroke and hyperlipidemia.

(Aging Clin Exp Res 2011; 23: 60-66)

๑2011, Editrice Kurtis

\section{INTRODUCTION}

Dementia is a serious health problem with a significant economic impact. Previous studies of populationbased cohort type have evaluated survival in relation to dementia, most reporting that the risk of death is higher in the presence of dementia than in its absence (1-6). A recent Danish population-based cohort study (14 years of follow-up) involving 3065 non-demented (73.7 \pm 6.8 years) and 234 demented $(83.3 \pm 7.0$ years) subjects at baseline, showed that the hazard ratio (HR) of death increased from 1.82 for very mildly demented to 9.52 for severely demented subjects (7). However, most studies have analyzed mortality in patients with cognitive impairment as a global diagnosis (7) or only in patients with Alzheimer's disease (AD) $(3,5)$. Only a few rare studies have considered mortality in other types of dementia, such as mixed dementia (AD plus vascular), which is very frequent in the very old, or in mild cognitive impairment $(\mathrm{MCI})(8,9)$. In addition, the non-demented subjects in these studies are often significantly younger $(2,3)$ and have significantly fewer comorbid conditions than the group of demented patients. Also, there are only a few studies examining short-term (1year) mortality and rare ones examining long-term (5year) mortality in acutely ill very old patients after discharge from hospital, and information on the same population remains scarce.

Key words: Aged, Alzheimer's disease, dementia, long term mortality, short-term mortality.

Correspondence: Dr. Dina Zekry, Rehabilitation and Geriatrics Department, Geneva University, 3 Chemin du Pont-Bochet, Thônex, Switzerland.

E-mail: dina.zekry@hcuge.ch

Received February 14, 2011; accepted in revised form February 25, 2011. 
We therefore studied the relationship between the various etiologies and severities of dementia and $\mathrm{MCI}$ and intra-hospital, short- and long-term mortality in a population of very old, acutely ill patients discharged from a geriatric hospital, in a prospective cohort study. We investigated the extent to which cognitive diagnosis was of greater added prognostic value in a population having the same number of other comorbid conditions.

\section{METHODS}

\section{Patients and data collection}

We carried out a prospective study in an acute 300bed geriatric hospital (HOGER), in which $22.7 \%$ of patients are admitted directly from the community, $54.0 \%$ are referred by the emergency unit, and $23.3 \%$ are transferred from other divisions of the university hospitals of Geneva, Switzerland. The patients and data collection have been described elsewhere $(10,11)$. Briefly, a representative sample of all consecutive admissions of patients aged 75 years and over in 2004 was selected by randomization, with a sampling fraction of $30 \%$ and a computer-generated randomization table. Exclusion criteria were disorders interfering with psychometric assessment (severe deafness or blindness, or major behavioral problems) and terminal illness. The local Ethics Committee approved the protocol, and patients, their families or legal representatives gave their signed informed consent. Patients' demographic data did not significantly differ from that for all patients admitted to HOGER over the same period. Our sample was therefore representative of all patients admitted to this hospital, demonstrating the reliability and quality of the randomization procedure used.

Medical history was recorded on a standardized form and the same geriatrician carried out a comprehensive geriatric assessment of all patients. Annual follow-up was carried out by the same assessment procedure, over a 5-year period.

\section{Socio-demographic data}

Data recorded included age, sex, native language, marital status, living conditions and educational level $(1=\leq 11 ; 2=12-14 ; 3=\geq 15$ years of schooling $)$.

\section{Cognitive diagnosis}

The same neuropsychologist assessed all subjects for clinical dementia, at least one week after admission, to avoid the effects of concomitant delirium. The MiniMental State Examination (MMSE) and the Short Cognitive Evaluation Battery (12) were used. According to screening results, the same neuropsychologist carried out a comprehensive standardized neuropsychological assessment, to determine the etiology and severity of clinical dementia, as previously described $(10,11)$. Briefly, the battery of neuropsychological tests included the fol- lowing specific tests of cognitive function: the Mattis Dementia Rating Scale as a global scale; Buschke Double Memory Test (16 or 48 items according to education level), which assesses episodic memory and provides cognitive support for both encoding and retrieval, discriminating effectively between normal elderly subjects and subjects with mild dementia; Trail-Making Test, which measures mental flexibility, and Verbal Fluency Test, which investigates verbal incitement, both these tests assessing executive function; Consortium to Establish a Registry for Alzheimer's Disease (CERAD) Figures, which measure visuospatial and construction abilities; Lexis or Bachy test for language, and Digit Symbol test for evaluating attention. Dementia severity was assessed on the Clinical Dementia Rating Scale (CDR) (13) [score 0.5 for MCI (14), score 1 for mild, score 2 for moderate and score 3 for severe dementia]. The formal clinical criteria used for diagnosis were those of the Diagnostic and Statistical Manual of Mental Disorders, 4th ed., text revision (DSM IV-TR) (15), the National Institute of Neurological and Communicative Diseases and Stroke/Alzheimer's Disease and Related Disorders Association (NINCDS-ADRDA) (16), and the National Institute of Neurological Disorders and Stroke - Association Internationale pour la Recherche et l'Enseignement en Neuroscience (NINDS-AIREN) (17). Brain imaging was also carried out and patients were then assigned to one of three groups: a) normal cognition, b) $\mathrm{MCI}$, and c) dementia of various types: $\mathrm{AD}$, vascular dementia $(\mathrm{VaD})$, mixed dementia $(\mathrm{MD})$, degenerative and vascular dementia in the same patient, and other types of dementia.

\section{Comorbidity}

From our previous work on this cohort (18), the best prognostic predictor of 5-year mortality was the Geriatric Index of Comorbidity (GIC) (19), class 4 multiplying the risk of death by 4 , so we used this comorbidity score in this study. The same geriatrician calculated the score for each patient, via an extensive review of the patient's medical records and administrative data for diagnoses, established at or before enrolment in this study and by standardized interviews with patients and/or surrogates. In computing the GIC, each of the 15 most prevalent clinical conditions (ischemic or organic heart disease, primary arrhythmias, heart disease with a non-ischemic or organic origin, hypertension, stroke, peripheral vascular disease, diabetes mellitus, anemia, gastro-intestinal disease, hepatobiliary disease, renal disease, respiratory disease, parkinsonism and nonvascular neurologic disease, musculoskeletal disorders, malignancies) is graded on a 0 to 4 disease severity scale, according to the following general framework: 0 = absence of disease, 1 = asymptomatic disease, 2 = symptomatic disease requiring medication but under satisfactory control, 3 = symptomatic disease uncon- 
trolled by therapy, and $4=$ life-threatening or the most severe form of the disease. The GIC classifies patients into four classes of increasing somatic comorbidity. Class 1 comprises patients who have one or more conditions with a disease severity grade $\leq 1$, class 2 patients who have one or more conditions with a disease severity grade of 2, class 3 patients who have one condition with a disease severity of 3 , other conditions having a disease severity $\leq 2$, and class 4 patients who have two or more conditions with a disease severity of 3 or one or more conditions with disease severity of 4 . Only $2 \%$ of patients were classified as class 1 , allowing us to combine classes 1 and 2 for purposes of analysis.

\section{Outcomes}

The outcomes of interest were intra-hospital mortality, death by December 31, 2005 for short-term mortality (1-year mortality) and December 31, 2009 for longterm mortality (5-year mortality). Information was obtained by yearly assessment, telephone calls to the patient, the patient's family and/or the patient's general practitioner. Mortality data were confirmed by the data of the population registry of the Canton of Geneva.

\section{Statistical methods}

We checked the normality of the data by carrying out skewness and kurtosis tests. Data for continuous variables are presented as means \pm 1 standard deviation (SD). Kruskal-Wallis tests were performed to compare the data for the following groups: cognitively normal patients, patients with $\mathrm{MCI}$, and demented patients. We first investigated the univariate relationship between each independent variable and intra-hospital, 1-year and 5-year mortality. We used Cox proportional hazards models to take into account the time to the event. The independent variables assessed as possible predictors included age, sex, cognitive diagnosis (normal, $\mathrm{MCI}$, or dementia), dementia etiology ( $\mathrm{AD}, \mathrm{VaD}$ and $\mathrm{MD}$; "other dementia" was excluded from the analysis due to its heterogeneity and small size) and dementia severity treated as a dichotomous variable (CDR 0.5-1 = mild; CDR 23 = moderate to severe dementia). We then entered all independent variables, together with intra-hospital, 1year and 5-year mortality as the dependent variable, in multiple Cox models. The hazard proportional assumption was respected for each regression. HR and their $95 \%$ confidence intervals (CI) were calculated. Pseudo R-squared $\left(\mathrm{R}^{2}\right)$, which provides information about the proportion of the variance explained by the model, was computed with the Stata "str2ph" command, based on Royston's modification of O'Quigley, Xu $\&$ Stare's modification of Nagelkerke's $\mathrm{R}^{2}$ statistic for proportional hazards models with censored survival data. Statistical analyses were performed with Stata software version 11.1, Texas, U.S.
Table 1 - Number of intra-hospital deaths, after 1- and 5-year follow-ups, according to cognitive impairment diagnosis.

\begin{tabular}{|c|c|c|c|c|c|c|}
\hline & \multicolumn{2}{|c|}{ Intra-hospital } & \multicolumn{2}{|c|}{ One year } & \multicolumn{2}{|c|}{ Five years } \\
\hline & $\mathbf{n}$ & $\%$ & $\mathbf{n}$ & $\%$ & $\mathbf{n}$ & $\%$ \\
\hline Non-demented & 12 & 54.5 & 39 & 37.5 & 115 & 43.7 \\
\hline $\mathrm{MCI}$ & 3 & 13.6 & 10 & 9.6 & 27 & 10.3 \\
\hline Demented & 7 & 31.9 & 55 & 52.9 & 121 & 46.0 \\
\hline $\mathrm{AD}$ & 1 & 4.5 & 16 & 15.4 & 45 & 17.1 \\
\hline $\mathrm{MD}$ & 5 & 22.7 & 27 & 26.0 & 50 & 19.0 \\
\hline $\mathrm{VaD}$ & 1 & 4.5 & 7 & 6.7 & 17 & 6.5 \\
\hline $\begin{array}{l}\text { Other types } \\
\text { of dementia }\end{array}$ & 0 & - & 5 & 4.8 & 8 & 3.0 \\
\hline $\begin{array}{l}\text { Total number } \\
\text { of deaths }\end{array}$ & 22 & 100 & 104 & 100 & 263 & 100 \\
\hline
\end{tabular}

MCI: mild cognitive impairment (14); AD: Alzheimer's disease; MD: mixed dementia; VaD: vascular dementia.

\section{RESULTS}

Of the 444 patients studied (mean age $85.3 \pm 6.7$; $74.0 \%$ women), 206 were cognitively normal, 48 had $\mathrm{MCI}$, and 190 had dementia: 75 cases of $\mathrm{AD}, 20$ of $\mathrm{VaD}, 82$ of $\mathrm{MD}$, and 13 of other types of dementia (3 cases of dementia with Lewy bodies, 3 of Parkinson's disease with dementia, 1 of Creutzfeld-Jacob disease, 1 of cortico-basal dementia, 3 of fronto-temporal dementia, 1 of hydrocephaly with normal pressure, and 1 of alcohol-related dementia). Of the 444 patients, 22 (5\%) died during hospitalization, 104 (23.4\%) died during the first year after discharge, and 263 died during the 5 years after discharge (59.2\%); 115 were cognitively normal (43.7\%); 121 demented (46\%) and $27 \mathrm{MCI}$ (10.3\%), with similar proportions for each group ( $p$ was not significant). Details are shown in Table 1.

The socio-demographic data and clinical features by cognitive impairment diagnosis have been published previously (11) (Table 2). Briefly, the groups compared (cognitively normal, $\mathrm{MCI}$ and demented) were similar in age, sex and education level. Patients without dementia were more likely to live alone $(65 \%)$ than demented patients (50\% of patients with MCI or dementia); $8 \%$ of demented patients were living in a nursing home, whereas this was the case for only $1 \%$ of non-demented patients. The GIC scores were very high; the majority of patients were assigned to GIC class 3 ( $n=310,69.8 \%)$, followed by class $4(n=91,20.5 \%)$ and classes $1-2(n=43,9.7 \%)$ and GIC scores did not differ significantly between patients with different cognitive status.

Patients in the VaD group tended to be younger and were more likely to be male, although these trends were not statistically significant. They had higher average comorbidity scores, when compared with other demented patients, more frequent hypertension, stroke and hyperlipidemia. Comorbidity did not increase with dementia severity. 
Table 2 - Patients' socio-demographic and clinical variables according to cognitive status.

\begin{tabular}{|c|c|c|c|c|c|c|c|}
\hline \multirow{2}{*}{$\begin{array}{l}\text { Characteristics } \\
\mathrm{Age}^{\mathrm{a}}\end{array}$} & \multicolumn{2}{|c|}{ Cognitively normal $(n=206)$} & \multicolumn{2}{|c|}{$\operatorname{MCI}(n=48)$} & \multicolumn{2}{|c|}{ Demented $(n=190)$} & \multirow{2}{*}{$\frac{\boldsymbol{p}_{\text {-value }}^{\text {c }}}{0.093}$} \\
\hline & 84.5 & \pm 7.0 & 85.0 & \pm 6.6 & 86.1 & \pm 6.4 & \\
\hline Female $^{\mathrm{b}}$ & 151 & $73.3 \%$ & 40 & $83.3 \%$ & 137 & $72.1 \%$ & 0.278 \\
\hline Educational level $^{\mathrm{a}}$ & & & & & & & 0.408 \\
\hline $1=\leq 11$ years & 123 & $59.7 \%$ & 31 & $64.6 \%$ & 103 & $54.2 \%$ & \\
\hline $2=12-14$ years & 66 & $32.0 \%$ & 10 & $20.8 \%$ & 66 & $34.7 \%$ & \\
\hline $3=\geq 15$ years & 17 & $8.3 \%$ & 7 & $14.6 \%$ & 21 & $11.1 \%$ & \\
\hline Living conditions ${ }^{\mathrm{a}}$ & & & & & & & 0.002 \\
\hline At home & 204 & $99.0 \%$ & 47 & $97.9 \%$ & 175 & $92.1 \%$ & \\
\hline Nursing home & 2 & $1.0 \%$ & 1 & $2.1 \%$ & 15 & $7.9 \%$ & \\
\hline GIC score $e^{\mathrm{a}}$ & & & & & & & 0.290 \\
\hline Classes 1 and 2 & 20 & $9.7 \%$ & 5 & $10.4 \%$ & 18 & $9.5 \%$ & \\
\hline Class 3 & 150 & $72.8 \%$ & 35 & $72.9 \%$ & 125 & $65.8 \%$ & \\
\hline Class 4 & 36 & $17.5 \%$ & 8 & $16.7 \%$ & 47 & $24.7 \%$ & \\
\hline Hypertension ${ }^{b}$ & 145 & $70.4 \%$ & 28 & $58.3 \%$ & 130 & $68.4 \%$ & 0.271 \\
\hline Ischemic heart disease $e^{b}$ & 54 & $32.0 \%$ & 13 & $31.7 \%$ & 41 & $25.8 \%$ & 0.442 \\
\hline Diabetes mellitus $^{\mathrm{b}}$ & 43 & $20.9 \%$ & 10 & $20.8 \%$ & 32 & $16.8 \%$ & 0.567 \\
\hline Stroke $e^{\mathrm{b}}$ & 20 & $11.8 \%$ & 4 & $9.8 \%$ & 33 & $20.8 \%$ & 0.047 \\
\hline Known dyslipidemiab & 27 & $16.0 \%$ & 7 & $17.1 \%$ & 23 & $14.5 \%$ & 0.889 \\
\hline
\end{tabular}

Univariate and multiple Cox proportional hazard modeling (Table 3)

The following outcomes are shown in Table 3: intrahospital mortality, mortality 1 year after discharge and 5 years after discharge, including all the potentially predictive variables tested: presence or absence of dementia, dementia etiology and dementia severity.

For the three outcomes, mortality was significantly

Table 3 - Univariate Cox regression predicting intra-hospital, 1- and 5-year mortality $(n=444)$.

\begin{tabular}{|c|c|c|c|c|c|c|c|c|c|c|c|c|}
\hline \multirow[b]{2}{*}{ Characteristics } & \multicolumn{4}{|c|}{ Intra-hospital } & \multicolumn{4}{|c|}{ One-year mortality } & \multicolumn{4}{|c|}{ Five-year mortality } \\
\hline & $\begin{array}{c}\text { Crude } \\
\text { HR }\end{array}$ & $95 \% \mathrm{CI}$ & $p$ & $\underset{\mathbf{R}^{2}}{\text { Pseudo }}$ & HR & 95\% CI & $p$ & $\begin{array}{c}\text { Pseudo } \\
\mathbf{R}^{2}\end{array}$ & HR & 95\% CI & $p$ & $\begin{array}{c}\text { Pseudo } \\
\mathbf{R}^{2}\end{array}$ \\
\hline $\mathrm{Age}^{\mathrm{a}}$ & 1.08 & $1.00-1.16$ & 0.029 & 0.027 & 1.07 & $1.04-1.15$ & $<0.001$ & 0.115 & 1.07 & $1.05-1.09$ & $\leq 0.001$ & 0.097 \\
\hline Male us female & 1.07 & $0.41-2.81$ & 0.888 & 0.001 & 1.27 & $0.83-1.93$ & 0.271 & 0.007 & 1.52 & $1.17-1.98$ & 0.002 & 0.021 \\
\hline Demented vs normal & 0.65 & $0.26-1.62$ & 0.353 & 0.009 & 1.54 & $1.05-2.26$ & 0.028 & 0.028 & 1.27 & $1.00-1.62$ & 0.053 & 0.009 \\
\hline $\begin{array}{l}\text { Demented } v \text { s } \\
\text { MCI us normal }\end{array}$ & & & & 0.005 & & & & 0.088 & & & & 0.009 \\
\hline Normal & 1.00 & - & - & & 1.00 & - & - & & 1.00 & - & - & \\
\hline $\mathrm{MCI}$ & 1.08 & 0.29-3.99 & 0.904 & & 1.07 & $0.54-2.16$ & 0.836 & & 0.99 & $0.65-1.50$ & 0.904 & \\
\hline Demented & 0.66 & $0.25-1.71$ & 0.389 & & 1.56 & $1.04-2.35$ & 0.033 & & 1.27 & $0.98-1.64$ & 0.068 & \\
\hline Type of dementia & & & & 0.021 & & & & 0.143 & & & & 0.016 \\
\hline Normal & 1.00 & - & - & & 1.00 & - & - & & 1.00 & - & - & \\
\hline $\mathrm{MCI}$ & 1.08 & $0.29-3.99$ & 0.904 & & 1.08 & $0.54-2.16$ & 0.832 & & 0.99 & $0.65-1.51$ & 0.962 & \\
\hline $\mathrm{AD}$ & 0.21 & $0.03-1.67$ & 0.142 & & 1.11 & $0.62-2.00$ & 0.719 & & 1.11 & $0.79-1.57$ & 0.548 & \\
\hline $\mathrm{VaD}$ & 0.81 & $0.10-6.58$ & 0.846 & & 1.96 & $0.87-4.37$ & 0.019 & & 2.00 & $1.18-3.33$ & 0.008 & \\
\hline $\mathrm{MD}$ & 1.06 & $0.36-3.09$ & 0.360 & & 1.80 & $1.10-2.94$ & 0.102 & & 1.22 & $0.87-1.70$ & 0.247 & \\
\hline Severity of dementia & & & & 0.005 & & & & 0.018 & & & & 0.015 \\
\hline Normal & 1.00 & - & - & & 1.00 & - & - & & 1.00 & - & - & \\
\hline CDR 0.5-1 & 0.63 & $0.22-1.83$ & 0.396 & & 1.14 & $0.71-1.83$ & 0.879 & & 1.04 & $0.78-1.39$ & 0.772 & \\
\hline CDR 2-3 & 0.91 & $0.31-2.67$ & 0.868 & & 1.93 & $1.22-3.04$ & 0.005 & & 1.47 & $1.09-1.98$ & 0.011 & \\
\hline
\end{tabular}

aNormalized by square root transformation. MCI: mild cognitive impairment (14); AD: Alzheimer's disease; VaD: vascular dementia; MD: mixed dementia; CDR: Clinical Dementia Rate (13); HR: hazard ratio; CI: confidence interval. Entries in bold type: significant results. 
associated with age, each additional year increasing the risk of death by $7-8 \%$. Being male increased the risk of death by a factor of 1.5 only for 5 -year mortality. For intra-hospital mortality, none of the independent variables was a predictor of the outcome. For this reason, we used only univariate models and not multiple models for this outcome. Dementia was associated with a significantly higher risk of 1-year death than not being demented ( $p=0.028$ ), increasing the risk by $50 \%$, although considering only the $\mathrm{MCI}$ group $(p=0.033)$. This association disappeared when the outcome was the risk of 5year death. VaD was the only type of dementia significantly associated with a double risk of death at 1- and 5year follow-ups. $\mathrm{MCI}, \mathrm{AD}$ and $\mathrm{MD}$ were not predictive of intra-hospital, short- or long-term mortality. Severely demented patients (CDR 2-3; regardless of etiology) had a $100 \%$ higher risk of dying than non-demented controls when 1-year death was the outcome and a $50 \%$ higher risk of dying when 5-year death was the outcome.

The introduction of all variables into the full model eliminated the association of moderate and severe dementia ( $\mathrm{HR}=1.7,95 \% \mathrm{CI}=0.57-5.14, p=0.334$ ) for 1 -year and (HR=1.2, 95\% Cl=0.61-2.27, $p=0.619)$ for 5-year mortality; particularly $\mathrm{VaD}: \mathrm{HR}=1.3,95 \% \mathrm{CI}=0.44-4.02$, $p=0.615$ for 1 -year and $\mathrm{HR}=1.7,95 \% \mathrm{CI}=0.86-3.30$, $p=0.125$ for 5 -year mortality. Age remained significantly associated with 1-year mortality ( $\mathrm{HR}=1.07,95 \%$ $\mathrm{CI}=1.03-1.11, p<0.001$ ), accounting for $12.5 \%$ of the variability of this outcome. Age $(\mathrm{HR}=1.08,95 \% \mathrm{Cl}=1.05$ $1.10, p<0.001)$ and $\operatorname{sex}(\mathrm{HR}=1.67,95 \% \mathrm{Cl}=1.21-2.30$, $p=0.002$ ) remained significantly associated with 5-year mortality, also accounting for $12.7 \%$ of the variability of this outcome.

\section{DISCUSSION}

This series of elderly inpatients (mean age 85 years) was representative of the overall population of patients in this geriatric hospital. The prevalence of dementia (43\%) was very high; the prevalence of dementia in elderly inpatients (acute geriatric wards) has been reported to be between 20 and $30 \%$. A previous study carried out in the same hospital reported a prevalence of $30 \%$. The difference between these two studies in the same hospital is statistically significant $(p=<0.001)(20)$. These findings probably reflect the systematic and complete assessment of cognitive impairment in the random sample used to determine the prevalence of dementia. The inclusion of a large number of patients with dementia in this study was useful, as it was possible to measure the impact of dementia on the outcomes of interest. In addition, comorbid scores were very high and similar in all three groups (cognitively normal, demented, $\mathrm{MCI}$ ) allowing comparisons and then assessment of the influence of dementia among other comorbidities.
In our population of very old patients, the 5-year mortality rate was almost $60 \%$ after discharge. This rate is similar to that reported in previous studies. Age itself is a well-known negative prognostic risk factor for death, and this factor accounted for almost $10 \%$ of the variance of the outcome in this study in the univariate model. In addition, in the multiple regression analysis, age was the only remaining predictor for 1 - and 5-year mortality risk.

For intra-hospital mortality, dementia, independent of etiology or severity, was not a predictor of death. Recently, a retrospective study based on hospital discharge database records in the period 1998-2003 from public hospitals in Andalusia, Spain, identified 40,482 cases of dementia, and reported that the intra-hospital mortality rate was greater (19.3 us 8.7\%) for patients with dementia compared with those without dementia. Dementia was an independent predictor of mortality [odds ratio $(\mathrm{OR})=1.77 ; 95 \% \mathrm{CI}=1.72-1.82$ ] (21). This study was conducted in general hospitals, all ages confounded. In an Italian study of 923 patients at least 65 years old (mean age $78.7 \pm 7.2,49 \%$ women) admitted to the acute care geriatric ward of an internal medicine department, in-hospital mortality was independently predicted by lower MMSE scores at hospital admission $(\mathrm{OR}=5.51,2.34-$ 12.9) (22). More recently, a study conducted in Iceland showed that moderate to severe cognitive problems predicted intra-hospital death but not death after 1 year of follow-up in a population of 749 patients discharged from an acute care hospital (23). In these studies, no complete systematic neuropsychological assessment was carried out, patients were classified with cognitive impairment as a global diagnosis, and etiology was not taken into account. In addition, the studied population was at least 10 years younger than ours.

In our study, $\mathrm{MCI}, \mathrm{AD}$ and $\mathrm{MD}$ were not predictive of short- or long-term mortality. In contrast, many studies have investigated survival as a function of dementia, and most have reported that the risk of death is higher in patients with dementia than in those without (3-9, 24-26). Most of these studies were population-based and examined survival from the time of dementia diagnosis, whereas our study, which was not designed for exactly the same purpose, was based on a selected group in a clinical setting and $42 \%$ of the cohort had already been diagnosed with dementia at baseline. In some of the above studies, cognition was assessed with the MMSE alone. The MMSE is neither sensitive nor specific for detecting dementia in a very old population and, in addition, does not distinguish between different types of dementia. One of the main strengths of our study was its standardized comprehensive assessment: the same neuropsychologist carried out the same systematic, complete neuropsychological assessment of all study patients, which increased the accuracy of cognitive diagnosis and allowed us 
to take the various etiologies and severity of dementia into account. Our study was also unusual in its inclusion of a group of patients with $\mathrm{MCI}$ in addition to the demented and non-demented groups. These patients behaved more like ones with normal cognition than demented patients. In addition, the same geriatrician scored the presence and extent of comorbidity in all patients.

In our study, features significantly associated with reduced survival at 1 and 5 years were being older, male, and having vascular or severe dementia. This effect can probably be explained by the higher average comorbidity scores in the $\mathrm{VaD}$ group compared with the $\mathrm{AD}$ and MD groups, especially more likely to be diagnosed as having cerebrovascular diseases such as stroke and cardiovascular risk factors like hypertension and high cholesterol. The multiple analyses confirmed these results: when all the variables were added in the multiple model, the effect of dementia severity and $\mathrm{VaD}$ completely disappeared, confirming that dementia (all etiologies) is not predictive of short- or long-term mortality, but the effect observed is probably linked to cerebro- and cardiovascular comorbidites (hypertension, stroke and hyperlipidemia).

Nonetheless, in the literature, higher comorbidity and poor functional status both had a negative effect on survival. Previous studies have also shown that poorer functional status before and at the time of hospital admission is associated with higher short- and long-term mortality and higher comorbidity scores (27-30).

Our study is subject to several limitations. First, as it focused on hospitalized elderly patients, it is difficult to generalize the conclusions drawn to all subjects, whether living in institutions or in the community. Second, this was a single-center study, and there is therefore a need to confirm the results obtained in other centers. Third, the enrolled patients were very old, acutely ill, and had a high burden of comorbidities.

In conclusion, we assessed intra-hospital, short-term and long-term mortality in acutely ill very old patients, with and without dementia. The groups with and without dementia were of similar age and had similar levels of comorbidity. $\mathrm{MCI}, \mathrm{AD}$ and $\mathrm{MD}$ were not predictive of intrahospital, short- or long-term mortality. Only VaD significantly predicted 1- and 5-year mortality. The $\mathrm{VaD}$ group had the highest comorbidity score. Comorbid medical conditions should therefore be considered more than cognitive impairment, when trying to predict intra-hospital, short- and long-term survival in very old medically ill inpatients. These findings have global implications for well-planned follow-up and home support after discharge, including medical and non-medical services.

\section{ACKNOWLEDGEMENTS}

We would like to thank the teams of Mrs. O. Baumer, L. Humblot and M. Cos for technical assistance. This work was supported by grant 3200B0-102069 from the Swiss National Science Foundation.

None of the authors received any consultancy fees or has any company holdings or patents. There are no conflicts of interest to report.

\section{REFERENCES}

1. Aevarsson O, Svanborg A, Skoog I. Seven-year survival rate after age 85 years: relation to Alzheimer disease and vascular dementia. Arch Neurol 1998; 55: 1226-32.

2. Bonsignore M, Heun R. Mortality in Alzheimer's disease. Dement Geriatr Cogn Disord 2003; 15: 231-6.

3. Larson EB, Shadlen MF, Wang L et al. Survival after initial diagnosis of Alzheimer disease. Ann Intern Med 2004; 140: 501-9.

4. Tschanz JT, Corcoran C, Skoog I et al. Cache County Study Group. Dementia: the leading predictor of death in a defined elderly population: the Cache County Study. Neurology 2004; 62: 1156-62.

5. Ganguli M, Dodge HH, Shen C, Pandav RS, DeKosky ST. Alzheimer disease and mortality: a 15-year epidemiological study. Arch Neurol 2005; 62: 779-84.

6. Rait G, Walters K, Bottomley C, Petersen I, Iliffe S, Nazareth I. Survival of people with clinical diagnosis of dementia in primary care: cohort study. BMJ 2010; 341: c3584.

7. Andersen K, Lolk A, Martinussen T, Kragh-Sørensen P. Very mild to severe dementia and mortality: A 14-year follow-up - The Odense study. Dement Geriatr Cogn Disord 2010; 29: 61-7.

8. Koedam EL, Pijnenburg YA, Deeg DJ et al. Early-onset dementia is associated with higher mortality. Dement Geriatr Cogn Disord 2008; 26: 147-52.

9. Guehne U, Angermeyer MC, Riedel-Heller S. Is mortality increased in mildly cognitively impaired individuals? A systematic literature review. Dement Geriatr Cogn Disord 2006; 21: 403-10.

10. Zekry D, Herrmann FR, Grandjean R et al. Demented versus nondemented very old inpatients: the same comorbidities but poorer functional and nutritional status. Age Ageing 2008; 37: 83-9.

11. Zekry D, Herrmann FR, Grandjean R et al. Does dementia predict adverse hospitalization outcomes? A prospective study in aged inpatients. Int J Geriatr Psychiatry 2009; 24: 283-91.

12. Robert PH, Schuck S, Dubois B et al; Investigators' Group. Screening for Alzheimer's disease with the short cognitive evaluation battery. Dement Geriatr Cogn Disord 2003; 15: 92-8.

13. Morris JC. The clinical dementia rating: Current version and scoring rules. Neurology 1993; 43: 2412-4.

14. Petersen RC, Smith GE, Waring SC, Ivik RJ, Tangalos EG, Kokmen E. Mild cognitive impairment: clinical characterization and outcome. Arch Neurol 1999; 56: 303-8.

15. American Psychiatric Association: Diagnostic and Statistical Manual of Mental Disorders, 4th ed., text revision (DSM IVTR). Washington: American Psychiatric Association, 2000.

16. McKahnn G, Drachman D, Folstein M, Katzman R, Price D, Stadlan EM. Clinical diagnosis of Alzheimer's disease: report of the NINCDS-ADRDA Work Group under the auspices of the Department of Health and Human Services Task Force on Alzheimer's Disease. Neurology 1984; 34: 939-44.

17. Roman GC, Tatemichi TK, Erkinjuntti T et al. Vascular dementia: diagnostic criteria for research studies: report of the NINDS-AIREN International Workshop. Neurology 1993; 43 : 250-60.

18. Zekry D, Hermont Loures Valle B, Esposito F et al. Prospective comparison of six comorbidity indices as predictors of 5 years post 
hospital discharge survival in the elderly. Rej Res 2010; 13: 675-82.

19. Rozzini R, Frisoni GB, Ferrucci L et al. Geriatric Index of Comorbidity: validation and comparison with other measures of comorbidity. Age Ageing 2002; 31: 277-85.

20. Herrmann F, Mermod J-J, Henderson S et al. Epidemiology of dementia in Geneva. In Michel J-P, Ed. Management of Aging. The University of Geneva Experience. Basel: Ed. Karger, 1999: 94-100.

21. Guijarro R, San Román CM, Gómez-Huelgas R et al. Impact of dementia on hospitalization. Neuroepidemiology 2010; 35: 101-8.

22. Marengoni A, Cossi S, De Martinis M et al. Adverse outcomes in older hospitalized patients: the role of multidimensional geriatric assessment. Aging Clin Exp Res 2003; 15: 32-7.

23. Jónsson PV, Noro A, Finne-Soveri $\mathrm{H}$ et al. Admission profile is predictive of outcome in acute hospital care. Aging Clin Exp Res 2008; 20: 533-9.

24. Noale M, Maggi S, Minicuci N, et al; ILSA Working Group. Dementia and disability: impact on mortality. The Italian Longitudinal Study on Aging. Dement Geriatr Cogn Disord 2003; 16: 7-14.
25. Helmer C, Joly P, Letenneur L, Commenges D, Dartigues JF. Mortality with dementia: results from a French prospective community-based cohort. Am J Epidemiol 2001; 154: 642-8.

26. Wolfson $\mathrm{C}$, Wolfson $\mathrm{DB}$, Asgharian $\mathrm{M}$ et al. A reevaluation of the duration of survival after the onset of dementia. N Engl J Med 2001; 344: 1111-6.

27. Covinsky KE, Justice AM, Rosenthal GE, Palmer R, Landefeld $\mathrm{CS}$. Measuring prognosis and case mix in hospitalized elders: the importance of functional status. J Gen Intern Med 1997; 12: 203-8.

28. Inouye SK, Peduzzi PN, Robison JT, Hughes JS, Horwitz RI, Concato J. Importance of functional measures in predicting mortality among older hospitalized patients. JAMA 1998; 279: 1187-93.

29. Bertozzi B, Barbisoni P, Franzoni S, Rozzini R, Frisoni GB, Trabucchi M. Factors related to length of stay in a geriatric evaluation and rehabilitation unit. Aging Clin Exp Res 1996; 8: 170-5.

30. Minicuci N, Maggi S, Noale $M$ et al. Predicting mortality in older patients. The VELCA Study. Aging Clin Exp Res 2003; 15: 328-35. 